\title{
Le parrainage francophone/francophile comme outil d'intégration académique, sociale et linguistique
}

\section{Hélène Knoerr}

\section{(2) OpenEdition Journals}

Electronic version

URL: http://journals.openedition.org/ripes/2446

DOI: $10.4000 /$ ripes.2446

ISSN: 2076-8427

Publisher

Association internationale de pédagogie universitaire

\section{Electronic reference}

Hélène Knoerr, «Le parrainage francophone/francophile comme outil d'intégration académique, sociale et linguistique », Revue internationale de pédagogie de l'enseignement supérieur [Online], 36(1) | 2020, Online since 12 April 2020, connection on 08 September 2020. URL : http:// journals.openedition.org/ripes/2446; DOI : https://doi.org/10.4000/ripes.2446

This text was automatically generated on 8 September 2020

Article L.111-1 du Code de la propriété intellectuelle. 


\title{
Le parrainage francophone/ francophile comme outil d'intégration académique, sociale et linguistique
}

\author{
Hélène Knoerr
}

\section{Introduction}

1 L'acte d'apprendre comporte une composante sociale. Ainsi, pour Vygotsky (1962), l'apprentissage est le résultat de l'intériorisation de l'interaction sociale. L'étymologie même renvoie au latin collaborare, soit travailler ensemble. Ainsi, apprendre s'inscrit dans une démarche collaborative : on peut apprendre avec et par les autres. Le concept de collaboration se décline selon de multiples modalités en fonction de "l'intensité du rapport entre les personnes qui travaillent ensemble, la hiérarchie des partenaires, ainsi que [d]es lieux et [d]es époques où ces phénomènes de socialisation ont lieu " (Weinberg, Knoerr et Gohard-Radenkovic, 2016, p. 254). L'enseignement constitue ainsi une forme de collaboration puisque enseignants et apprenants participent ensemble à la construction des connaissances et des compétences de ces derniers, à l'intérieur d'une relation hiérarchique, verticale, les enseignants, détenteurs du savoir et de l'expertise, ayant un statut supérieur aux apprenants.

2 Cependant, une collaboration moins hiérarchique est possible : la collaboration entre étudiants. "Ce système renouvelle le schéma classique de la relation de formation où celui qui sait est debout, face à celui qui ne sait pas, qui est assis. [...] Les deux partenaires ne se font plus face mais se retrouvent côte à côte dans une relation d'accompagnement et de connivence, chacun apprenant de la situation » (Floor, 2010, p. 3). Même si les compétences linguistiques sont inégales, ils sont dans une relation de statut égalitaire - d'apprenant à apprenant. S'il y a relation hiérarchique entre 
professeur et étudiant, il n'y en a pas en revanche pas entre étudiant et étudiant puisqu'il n'y a pas de rapport de pouvoir entre eux.

Nous avons voulu expérimenter ce type de collaboration auprès de jeunes Canadiens anglophones issus de programmes de français langue seconde à l'école secondaire et arrivant en première année d'université dans le Régime d'immersion en français de l'Université d'Ottawa, la plus grande université bilingue en Amérique du Nord. Dans le Régime d'immersion en français, les étudiants anglophones suivent 42 crédits de leur programme en français, des cours de disciplines non linguistiques enseignés par des professeurs francophones et suivis majoritairement par des étudiants francophones (Knoerr, 2010). Il s'agit alors de jumeler des étudiants des deux groupes linguistiques suivant un même cours en français, les francophones servant de parrains aux anglophones (Weinberg, 2013), afin de faciliter l'intégration de ces derniers (Séror et Lamoureux, 2014). Ainsi, l'objectif est non seulement linguistique et culturel mais aussi académique.

4 Dans un premier temps, nous situerons notre étude dans son cadre théorique, celui du constructivisme, et définirons les concepts clés tels que collaboration et apprentissage collaboratif. Ensuite, nous décrirons le contexte pédagogique de l'étude, le Régime d'immersion en français de l'Université d'Ottawa, et plus précisément le dispositif de parrainage au cœur de cette recherche. Le protocole expérimental, les instruments et les participants feront l'objet de la section suivante. Les résultats seront présentés dans la quatrième partie et discutés dans la cinquième, afin de déterminer si les buts de l'expérience ont été atteints: (1) améliorer la réussite académique des étudiants du Régime d'immersion en français; (2) favoriser leur intégration sociale dans la communauté étudiante; (3) améliorer leurs compétences linguistiques en français. L'article conclura sur un bilan de l'expérience de parrainage et proposera quelques pistes de développement et d'amélioration pour ce type de dispositif.

\section{Cadre théorique : réussite académique, soutien social et apprentissage collaboratif}

Comme le résument De Ketele et Pirot (2000), «la réussite des études exige non seulement la capacité de répondre efficacement aux exigences universitaires, mais aussi de surmonter le déséquilibre provoqué par le passage d'un environnement connu (le milieu familial et l'enseignement secondaire) à un environnement de vie inconnu (le campus et les études universitaires) » (p. 367). Si cette transition est difficile pour les étudiants en général, elle l'est encore davantage pour ceux qui poursuivent leurs études supérieures dans leur langue seconde. Ainsi, pour réussir, il leur faut non seulement s'approprier la culture et les codes de l'université mais aussi la culture et les codes de l'autre communauté linguistique (Coulon, 1997). C'est à ce niveau que les dispositifs d'accompagnement ont une importance cruciale (Michaut, 2000; Romainville, 2000). Le soutien social ou réseau social, c'est-à-dire l'évaluation par un individu de l'aide apportée par son entourage (Procidiano et Heller, 1983; Sarason, Sarason, Potter III et Antoni, 1985) et la satisfaction découlant de la qualité perçue de cette aide (Sarason, Levine, Basham et Sarason, 1983), joue également un rôle majeur dans la réussite académique. Plusieurs études indiquent que les étudiants bénéficiant d'un important soutien social amical ont de meilleurs résultats (Demetriou, Goalen et Rudduck, 2000). 
6 Dans le cadre spécifique du Régime d'immersion en français, les obstacles rencontrés par les étudiants sont bien documentés (Séror et Lamoureux, 2014): en plus des difficultés linguistiques, ils ressentent «un manque d'accueil de la part de la communauté francophone à laquelle ils essaient de s'introduire en participant au Régime d'immersion en français» (p. 105). C'est pourquoi un dispositif d'accompagnement impliquant un apprentissage collaboratif nous a paru particulièrement indiqué pour venir en aide à cette population dont le nombre va croissant à l'Université d'Ottawa (plus de 2000 étudiants à ce jour).

7 Lebow (1991) arrime l'apprentissage collaboratif à sept piliers du constructivisme : collaboration, autonomie, réflexivité, générativité, engagement actif, pertinence personnelle et pluralisme. L'apprentissage collaboratif est par nature constructiviste : c'est un processus mental, interne et individuel qui se nourrit d'un processus de groupe permettant l'élaboration de représentations par le biais d'interactions humaines. Dans une conception constructiviste, l'apprentissage est le fruit d'échanges, de confrontations de points de vue (Jonassen, Davidson, Collins, Campbell et Haag, 1995; von Glaserfeld, 1995).

8 Selon les sociocognitivistes, l'apprentissage est la résultante d'une combinaison de trois facteurs principaux: l'interdépendance entre l'apprenant, l'environnement d'apprentissage et le milieu culturel; l'ancrage social et le transfert des connaissances. Placé dans des situations pédagogiques réelles, l'apprenant observe, donne sens, puis s'approprie peu à peu les actions des experts (Lave et Wenger, 1991; Wilson et Cole, 1991), dont le rôle diminue au fur et à mesure que la compétence des apprenants augmente. Grâce à des travaux en groupes, l'apprenant est auteur actif de son apprentissage et co-acteur de celui des autres (Brown et Palincsar, 1989; Collins, Brown et Newman, 1989; Cranton, 1984; Garrison, 1995).

9 Collaboration et coopération se situent sur un même continuum mais se distinguent par le degré d'autonomie et de contrôle des apprenants (Baudrit, 2007a, 2007b). Selon Springer (2010, p. 518), « la collaboration consiste en la participation à des activités collectives pour accomplir à plusieurs un but partagé». De plus, une situation collaborative met en présence des acteurs qui « (i) sont à peu près au même niveau et peuvent produire les mêmes actions, (ii) poursuivent un but commun et (iii) travaillent ensemble » (Dillenbourg, 1999 p. 9). Pour cet auteur la collaboration implique donc une certaine symétrie au sein des groupes : les membres se perçoivent comme égaux, de statut identique, et contribuent de manière équivalente.

10 En outre, la coopération repose sur la répartition des tâches entre les membres du groupe, chacun réalisant sa part individuelle pour que le but commun soit atteint (Johnson et Johnson, 1990). Par contre, dans un contexte de collaboration, chaque membre travaille individuellement à l'accomplissement des tâches liées à l'atteinte du but en apportant son éclairage personnel, sa contribution, à chacune des composantes, et participe à la co-construction de la tâche, à la co-réalisation de l'objectif.

11 Notre initiative est donc clairement collaborative :

- Le degré d'autonomie des apprenants est très important et ils contrôlent eux-mêmes leur apprentissage. Le rôle du professeur se borne à mettre les deux groupes en présence et à faire un suivi; les étudiants constituent eux-mêmes les paires ou groupes de parrainage, planifient l'horaire des rencontres et décident des contenus. Les étudiants d'immersion et les étudiants francophones sont de statuts identiques : étudiants de première année dans un cours d'introduction à la science politique. Par contre, leurs rôles sont différents: les 
francophones aident les francophiles dans la compréhension du cours de discipline en français. Ainsi, malgré une asymétrie linguistique de fait, le parrainage « se fait par les pairs et entre les pairs» (Weinberg, 2013, p. 109), les francophiles participant aussi activement que les francophones à la construction de la relation et de l'apprentissage de la matière du cours de discipline.

- Les apprenants contribuent individuellement à l'appropriation des contenus du cours de discipline et à la réalisation des tâches d'apprentissage. Chacun assiste aux cours magistraux, prend des notes, lit les textes, fait les travaux. Les groupes de parrainage révisent par la suite les notes de cours, décortiquent les textes de lecture, préparent les questions d'examen et les discussions des groupes de travaux dirigés. Mais chacun reçoit une note distincte dans le cours de discipline.

\section{Contexte pédagogique}

12 L'Université d'Ottawa, établissement bilingue, offre ses cours et la majorité de ses programmes de premier cycle tant en français qu'en anglais. Environ le tiers des étudiants est inscrit dans un programme en français, contre deux tiers dans des programmes en anglais, et on trouve également quelques étudiants qui suivent certains de leurs cours dans leur langue seconde. La particularité du Régime d'immersion en français est d'institutionnaliser ce fait en créant une structure d'accueil pour les étudiants francophiles. Un francophile est défini comme un étudiant « qui vient de la communauté anglophone mais qui apprécie vivre dans la culture et dans la langue française" (Knoerr, 2016, p.50). Ceux-ci ont également la possibilité de suivre parallèlement à leurs cours de discipline en français des cours d'encadrement linguistique dont le but est de les aider avec les difficultés d'ordre linguistique rencontrées dans le cours de discipline ${ }^{1}$.

Dans le cadre du Régime d'immersion en français, le jumelage entre francophones et francophiles a été opérationnalisé pour la première fois par Weinberg (2013) sous l'étiquette de "parrainage ", qui "s'appuie sur le concept d'échanges linguistiques où deux personnes de langues maternelles différentes travaillent ensemble et peuvent ainsi apprendre la langue de l'autre tout en découvrant sa personnalité et sa culture. Il est basé sur deux grands principes : celui de la réciprocité et celui de l'autonomie » (p. 109). Le principe de réciprocité s'entend dans le sens donné par Pléty (1996) : «Ce que les élèves peuvent s'apporter les uns aux autres » (p.153). En effet, même s'il est clair que les francophiles bénéficient des compétences linguistiques des francophones, les deux groupes contribuent également à la compréhension mutuelle de la matière du cours de discipline, dans lequel ils sont tous novices. Le principe d'autonomie a été développé dans la section précédente.

Le parrainage tel qu'entendu par Weinberg (2013) s'opère entre étudiants de même niveau académique - ils sont inscrits dans les mêmes cours de discipline - et de même statut - ils sont étudiants dans un cours de première année d'université - mais de compétences linguistiques différentes. Ce parrainage poursuivait un triple objectif : (1) améliorer l'expérience universitaire ${ }^{2}$ des anglophones qui suivent des cours de discipline en français en favorisant leur intégration dans la communauté étudiante, (2) renforcer leurs chances de réussite académique et (3) développer leurs compétences linguistiques. 

professeure d'histoire et une professeure de cours d'encadrement linguistique. Elles ont corédigé le projet, recruté et jumelé les participants. Pendant le trimestre, les étudiants se sont rencontrés pour discuter des difficultés éprouvées dans le cours d'histoire, ainsi que pour préparer leurs examens et leurs travaux pour ce cours. Le suivi a été assuré d'une part par la professeure de langue, qui vérifiait que les équipes se rencontraient régulièrement, et d'autre part par la professeure de discipline, qui communiquait régulièrement avec les étudiants et a organisé quelques rencontres. Le parrainage a connu un grand succès auprès des étudiants qui y ont participé, tant sur le plan de la réussite dans le cours de discipline que sur celui de la qualité de l'expérience humaine (Weinberg, 2013). Les participants (huit francophiles) ont cité, parmi les bénéfices retirés de l'expérience, l'amélioration des connaissances linguistiques (en particulier le vocabulaire et les habiletés productives), une meilleure compréhension du cours de discipline et une meilleure compréhension de l'autre groupe linguistique.

Nous avons repris et adapté cette expérience, afin d'en élargir la portée tout en offrant à d'autres étudiants la possibilité de profiter des avantages mis en évidence lors de sa première et seule occurrence. Nous avons appliqué les recommandations formulées par les participants à la suite de cette expérience : (1) commencer le parrainage très tôt dans la session et (2) organiser une rencontre initiale entre francophones et francophiles (Weinberg, 2013). Nous avons offert la possibilité aux étudiants anglophones de première année inscrits dans le Régime d'immersion en français d'être associés à des étudiants francophones qui suivent le même cours d'introduction à la science politique qu'eux. Le but de l'initiative était triple: (1) améliorer leur réussite académique; (2) favoriser leur intégration sociale dans la communauté étudiante; (3) améliorer leurs compétences linguistiques en français. Nous avons proposé cette option au cours de trois sessions universitaires (une session dure treize semaines). Nous présentons ici les perceptions des étudiants de ces trois cohortes relativement aux objectifs de ce programme de parrainage francophone/ francophile et nous tirons les conclusions de cette expérience.

\section{3. Étude}

Nous avons repris le concept initial et nous l'avons mis en œuvre avec quelques différences :

- Les rencontres de parrainage ont commencé la troisième semaine de cours (la session en compte 13);

- Le/ la professeure de discipline n'était pas impliqué/e dans le projet, si ce n'est pour en permettre la présentation pendant son cours ${ }^{3}$;

- La taille des groupes de parrainage était variable (de deux à six);

- La mise en relation entre parrains et parrainés s'est faite en fonction des affinités et des disponibilités de ceux-ci et non par décision des professeurs;

- La professeure du cours d'encadrement linguistique a organisé une rencontre initiale avec les participants;

- Le suivi s'est fait sous forme d'une rencontre avec la professeure du cours d'encadrement linguistique et les participants (première session) et de questionnaires (les trois sessions).

Revue internationale de pédagogie de l'enseignement supérieur, 36(1) | 2020 


\subsection{Protocole}

- Le recrutement des participants s'est fait pendant le cours de discipline : au début du cours, la professeure du cours d'encadrement linguistique a résumé le projet et a invité les francophones intéressés à prendre contact avec elle; elle a également souligné qu'ils pouvaient faire valider les heures consacrées au parrainage dans le cadre du programme de bénévolat de l'Université d'Ottawa, ce qui constituait un attrait. Elle a détaillé le projet avec les étudiants d'immersion pendant le cours d'encadrement linguistique. Un document expliquant la démarche a été affiché dans le campus virtuel des deux cours (voir annexe 1).

- Une première rencontre a été organisée au début de la troisième semaine de cours avec les francophones et les francophiles. La professeure du cours d'encadrement linguistique y a à nouveau expliqué le projet et ses objectifs et a suggéré diverses configurations (en paires, en petits groupes avec un ou plusieurs francophones pour un ou plusieurs francophiles). Elle a également proposé divers types d'activités - étudier ensemble, partager les notes de cours, discuter de la discipline ou de sujets divers - en insistant sur la nécessité d'utiliser uniquement le français. Les participants ont fait connaissance et, sur la base de la compatibilité de leurs emplois du temps, ont constitué les groupes en fonction de leurs préférences (paires ou groupes plus ou moins grands avec plus ou moins de francophiles), échangé leurs coordonnées et fixé un calendrier de rencontres.

- Au début de la première session (hiver 2015), six participants francophiles se sont portés volontaires. Ils étaient à nouveau six à la session d'hiver 2016 et 21 au cours de la session d'automne 2016, soit un total de 33 francophiles.

- À la fin de la session, tous les francophiles ont été invités de vive voix et par courriel à remplir un questionnaire en ligne pour faire l'évaluation de leur expérience de parrainage. Le questionnaire a été disponible pendant un mois et deux rappels ont été envoyés4.

- Trois francophiles de la session d'hiver 2015 (dorénavant H2015), trois francophiles de la session d'hiver 2016 (dorénavant H2016) et douze francophiles de la session d'automne 2016 (dorénavant A2016) ont répondu au questionnaire, soit au total 18 répondants.

\subsection{Participants}

Tous les francophiles étaient des étudiants canadiens (majoritairement de l'Ontario) issus des programmes de français langue seconde ou d'immersion française. Ils étaient tous étudiants de première année inscrits dans un cours d'introduction à la science politique : à l'hiver 2015 et l'hiver 2016 Politique et mondialisation et à l'automne 2016 Introduction à la science politique.

Il s'agissait de $80 \%$ de femmes et $20 \%$ d'hommes, âgés entre 17 et 19 ans, provenant pour la plupart de l'immersion française. Presque tous suivaient un programme en sciences sociales ou en arts. Cet échantillon reflète la composition démographique des 
étudiants du Régime d'immersion en français : les deux-tiers proviennent des programmes d'immersion au secondaire, contre un tiers des programmes de français de base et français enrichi ${ }^{5}$; la majorité est de sexe féminin; ils sont majoritairement inscrits en sciences humaines (dans l'ordre, sciences sociales, sciences de la santé, arts et administration).

\subsection{Instrument}

Le questionnaire développé à l'aide de SurveyMonkey (voir annexe 2) comportait dix items - huit questions à choix multiples, une échelle de gradation et une question ouverte (commentaires). Les questions portaient sur les attentes des participants envers le programme, la logistique des rencontres, leurs contenus (académique, social, linguistique) et la langue utilisée. Enfin, une question portait sur le niveau global de satisfaction envers le parrainage et une autre demandait d'identidier spécifiquement les bénéfices retirés, pour nous permettre de voir s'ils correspondaient aux objectifs escomptés.

\section{Résultats}

La première question cherchait à identifier les raisons qui ont poussé les étudiants à participer au parrainage. Si on regarde le nombre total de participants quelle que soit la cohorte (18 francophiles), il apparait que l'expérience linguistique est citée 16 fois comme motivation première, l'expérience académique et l'expérience sociale 12 fois (voir Figure 1). Ces motivations se retrouvent dans le même ordre si l'on ventile les réponses par cohorte: l'expérience linguistique est la première raison pour tous les répondants de $\mathrm{H} 2015$ et pour tous sauf un en H2016 et A2016; l'expérience académique et l'expérience sociale suivent à égalité pour tous les répondants de H2015 et pour les deux-tiers de ceux de H2016 et A2016.

Figure 1 : Répartition des réponses à la question 1- Pour quelle(s) raison(s) avez-vous participé au parrainage?

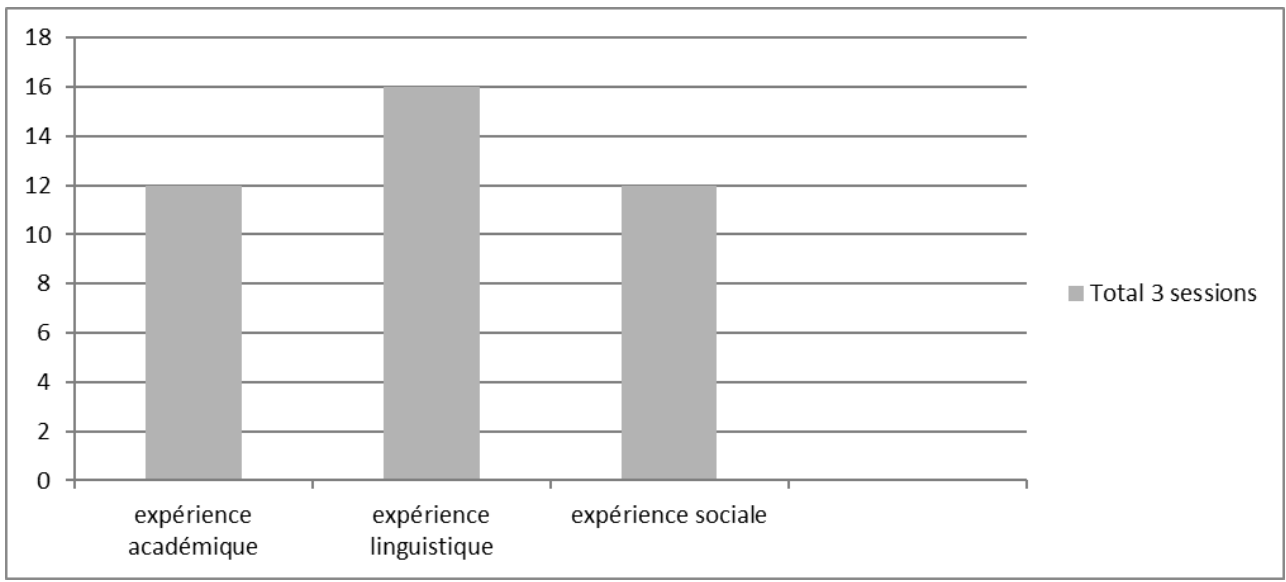

La deuxième question portait sur le nombre des rencontres de parrainage au cours de la session. La quasi-totalité des groupes se sont rencontrés au moins six fois en douze semaines, mais les chiffres varient considérablement entre les cohortes: plus de la moitié des étudiants de la session H2015 n'a eu qu'une ou deux rencontres alors que 
lors des sessions H2016 et A2016 plus de la moitié des groupes se sont rencontrés onze fois et plus. En réponse à la troisième question, la plupart des rencontres avaient lieu indépendamment du cours de discipline, généralement en semaine et parfois les fins de semaine (sauf pour H2015). Elles se tenaient de préférence dans une salle d'étude ou de classe (57\%, pour H2016) ou ailleurs sur le campus ( $80 \%$ pour H2015, $43 \%$ pour $\mathrm{H} 2016$ et $64 \%$ pour A2016) selon leurs réponses à la cinquième question.

En ce qui concerne le contenu des rencontres (cinquième question), les trois cohortes consacraient la majorité de leur temps à la révision des notes du cours de discipline, à des discussions personnelles et sociales et à des discussions sur le cours. Les travaux pour le cours de discipline, l'étude des textes à lire pour la semaine et des discussions spécifiques sur la langue française ont occupé le reste du temps (voir Figure 2).

Figure 2 : Réponses à la question 5 - Que faisiez-vous pendant vos rencontres de parrainage?

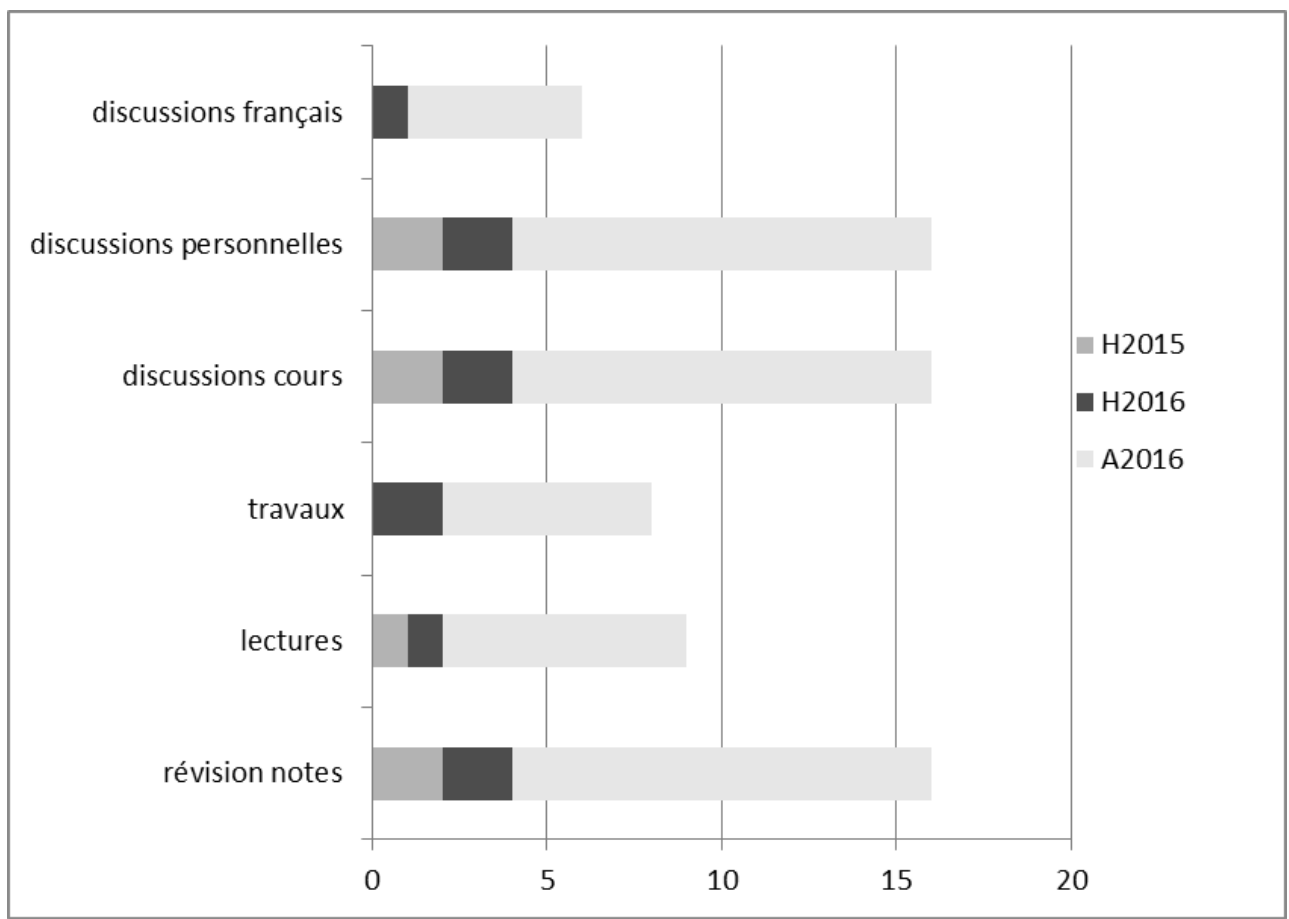

La sixième question voulait établir les langues utilisées au cours des rencontres. Le français a généralement été la langue du parrainage, conformément aux consignes données, consigne respectée par $100 \%$ des répondants de $\mathrm{H} 2015$ et les trois-quarts de ceux de A2016 mais seulement par la moitié de ceux de H2016.

La septième question portait sur l'évaluation du parrainage par les participants. L'expérience est jugée de manière positive par les trois cohortes (voir Figure 3). Le taux de satisfaction est le plus faible pour H2015 et le plus élevé pour H2016 (voir Figure 4). 
Figure 3 : Réponses globales à la question 7 - Sur une échelle de 0 à 5, comment évaluez-vous votre expérience de parrainage?

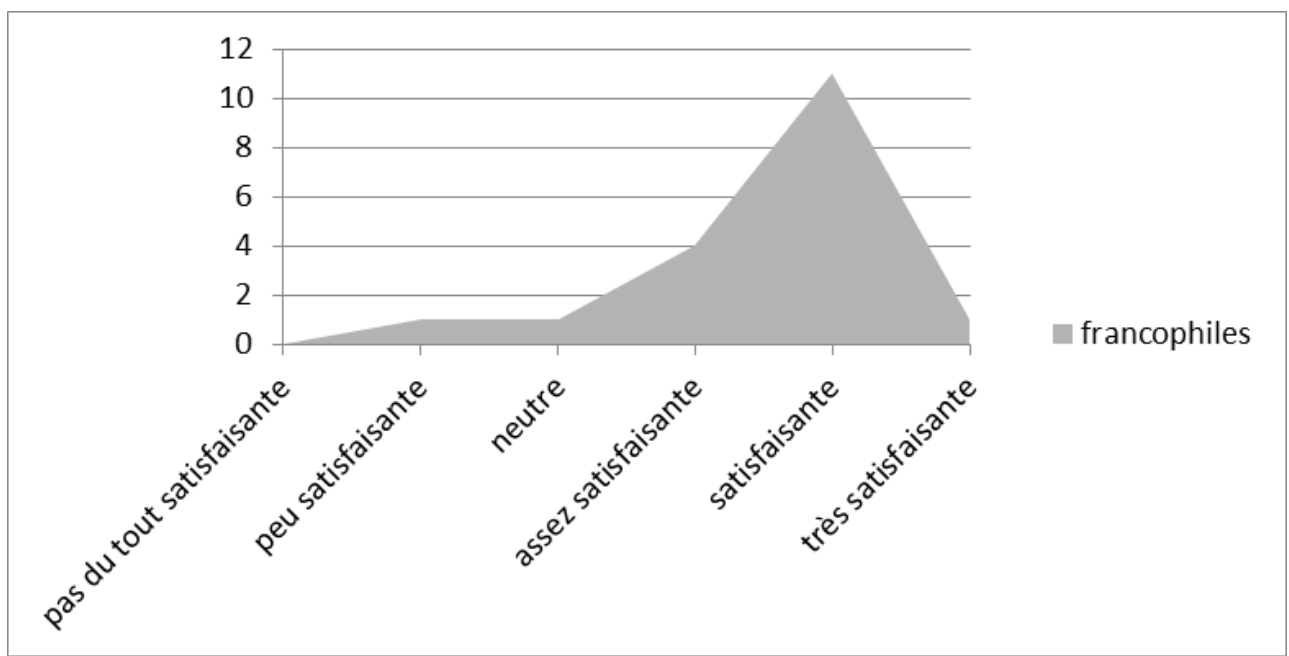

Figure 4 : Réponses par cohorte à la question 7 - Sur une échelle de 0 à 5 , comment évaluez-vous votre expérience de parrainage?

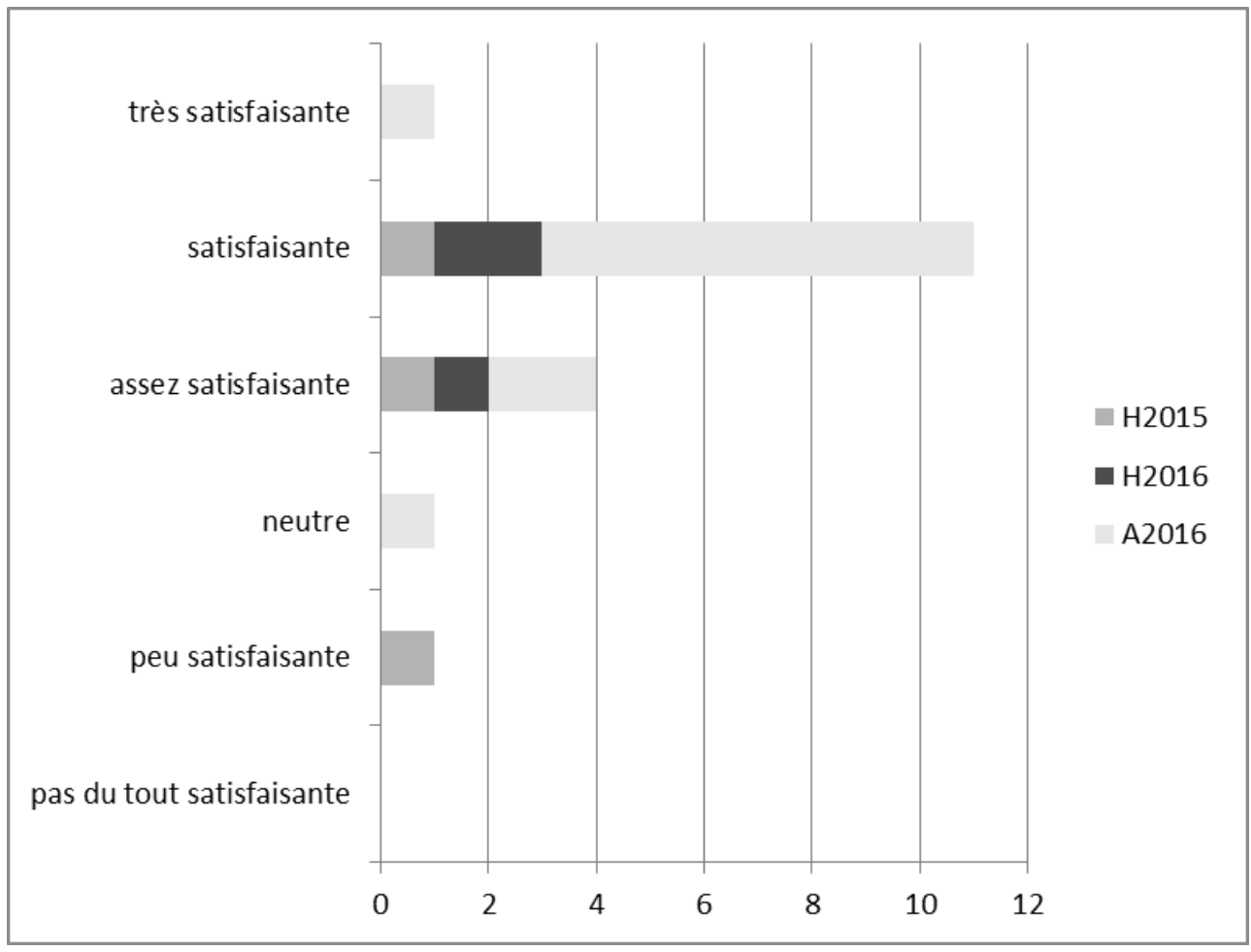

La huitième question voulait identifier le type d'organisation le plus apprécié dans la mise en place des groupes de parrainage. Le format préféré des trois cohortes est les petits groupes (à plus de $85 \%$ ), cette formule ayant été plébiscitée par H2015 qui n'a pourtant connu que le tandem.

Enfin, parmi la liste de bénéfices retirés de l'expérience de parrainage (neuvième question), les trois cohortes s'accordent pour dire que le principal est d'ordre linguistique (amélioration du français), le second d'ordre social (se faire de nouveaux 
amis) et humain (compréhension de l'autre groupe linguistique) et le troisième d'ordre académique (amélioration des connaissances dans la discipline) (voir Figure 5).

Figure 5 : Réponses globales à la question 9 - Quels bénéfices avez-vous retirés de votre expérience de parrainage?

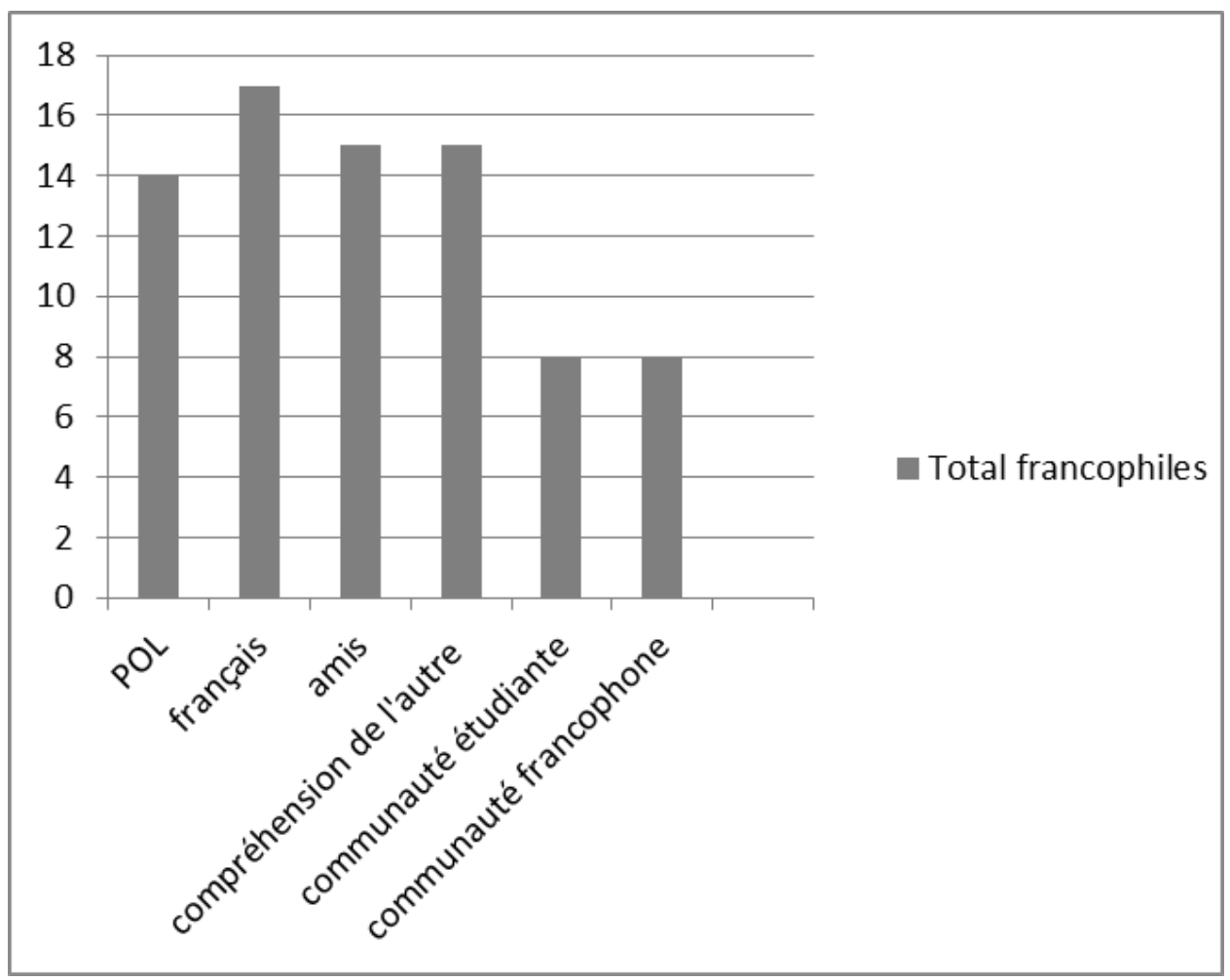

Si l'on ventile les réponses par cohortes, les réponses sont sensiblement les mêmes (voir Figure 6). 
Figure 6 : Réponses par cohortes à la question 9 - Quels bénéfices avez-vous retirés de votre expérience de parrainage?

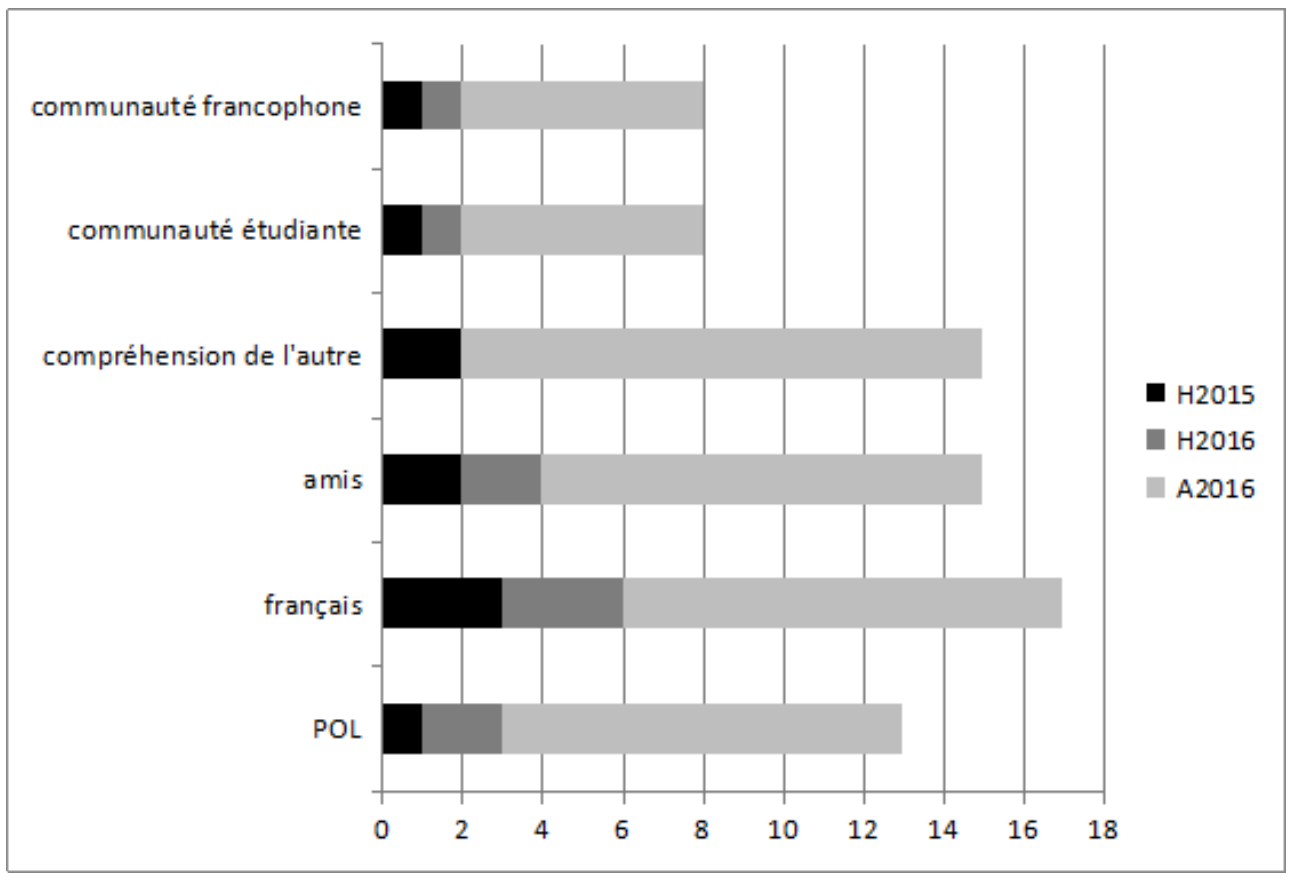

Les quelques participants qui ont fait des suggestions pour améliorer le programme (dixième question) ont souhaité davantage de structure et d'encadrement par la professeure du cours d'encadrement linguistique, y compris au niveau des activités les plus susceptibles de favoriser l'échange :

- Francophile 1: faire beaucoup d'activités (musées, journée étude, journée pizza, cinémas, etc.); rencontre faisant partie d'activités (on se rencontre et ensuite on fait une activité comme manger de la pizza, etc.);

- Francophile 2 : Plus de suggestions d'activités à faire et aussi de direction pour les parrains en ce qui concerne comment ils peuvent aider les francophiles.

À cet égard il est intéressant de noter le commentaire d'un étudiant francophone dans son évaluation de l'enseignement à la fin de la session : « Il serait intéressant de donner des indications [pour] que les tuteurs puissent programmer des activités cohérentes avec les demandes qui sont faites aux étudiants francophiles ».

\section{Discussion}

Le but du parrainage était triple : (1) améliorer la réussite académique des étudiants du Régime d'immersion en français; (2) favoriser leur intégration sociale dans la communauté étudiante; (3) améliorer leurs compétences linguistiques en français. Selon les participants, a-t-il été atteint? Nous ne pouvons baser notre discussion que sur les réponses des francophiles au questionnaire. Il n'a en effet pas été possible, faute de temps et de moyens, d'organiser des sessions de discussion ou des focus groups pour approfondir ou clarifier certains éléments. Cependant, on peut avancer un certain nombre de réponses. 


\subsection{Améliorer la réussite académique} est qu'ils se sont faits de nouveaux amis et qu'ils ont acquis une meilleure compréhension de l'autre groupe linguistique, deux aspects à forte teneur sociale. L'intégration à la communauté étudiante et à la communauté francophone sont par ailleurs mentionnées huit fois chacune (pour un total de 16 participants). On peut donc répondre affirmativement à la deuxième question, qui demandait si le programme favorisait leur intégration sociale dans la communauté étudiante.

\subsection{Améliorer les compétences linguistiques en français}

Le parrainage est certainement perçu comme un outil pour l'amélioration des compétences linguistiques en français, qui arrive en première place dans le palmarès des bénéfices retirés, surtout pour les étudiants de la session d'hiver 2015. C'est le premier bénéfice déclaré par les francophiles et c'était aussi leur motivation première pour participer à l'expérience.

\section{Conclusion}

Ayant repris l'expérimentation initiée par Weinberg (2013), nous avons conservé la terminologie d'origine. Nous sommes consciente du fait que le choix du terme " parrainage ", dans son acception générale et non dans celle de Weinberg (2013), peut prêter à confusion quant à la nature de la relation entre francophones et francophiles. Les francophones n'étaient pas perçus comme experts dans la discipline mais comme débutants, au même titre que les francophiles; ils voulaient aider ces derniers dans leur apprentissage linguistique, la langue, et non la discipline, étant le lieu de leur expertise en tant que locuteurs natifs mais pas en tant que linguistes ou pédagogues. L'expérience a montré que, selon les perceptions des francophiles, les deux groupes ne se considèrent pas en relation asymétrique mais réciproque, chacun bénéficiant des apports de l'autre. Un terme tel que « jumelage » serait peut-être plus adapté, bien qu'il implique une relation d'individu à individu et non de groupe à groupe. D'autre part, il faudrait décrire explicitement aux deux publics ce qu'ils peuvent retirer de cette expérience - le parrainage ne bénéficie pas seulement aux francophiles, les francophones en retirent également des avantages (ils peuvent recevoir une attestation de bénévolat, par exemple).

Nous avons également signalé la petite taille de l'échantillon, 18 francophiles, ce qui est supérieur à l'échantillon de Weinberg 2013 (huit francophiles) et indiqué la limite que

Revue internationale de pédagogie de l'enseignement supérieur, 36(1) | 2020 
constitue l'absence de sessions de discussion ou de focus groups pour revenir sur les réponses au questionnaire et les approfondir. étudiants du Régime d'immersion en français perçoivent le parrainage comme un moyen d'améliorer leurs compétences linguistiques et académiques et de favoriser leur intégration sociale. De plus, le parrainage semble permettre un rapprochement entre les deux communautés linguistiques et culturelles, selon $41 \%$ des participants. En effet, la compréhension de l'autre communauté linguistique arrive au deuxième rang des bénéfices déclarés par les francophiles, au même titre que le gain de nouveaux amis. La situation préférée par la majorité des répondants consiste en une configuration en petits groupes qui se rencontrent une fois par semaine, peu importe où, pour réviser les notes prises pendant le cours et avoir des discussions d'ordre personnel ou social ainsi que des discussions sur le cours, en français. Un encadrement pédagogique fourni par le professeur permettrait aux francophones de savoir quelles activités proposer aux francophiles afin d'optimiser l'apprentissage linguistique.

Nos résultats rejoignent ici ceux de Weinberg (2013) : les francophiles ayant participé au parrainage ont indiqué qu'ils en avaient retiré une amélioration des connaissances linguistiques (5 sur 8), une meilleure compréhension du cours de discipline (6 sur 8), et «ils ont aussi été satisfaits d'avoir des liens amicaux, de faire des échanges culturels » (Weinberg, Knoerr et Gohard-Radenkovic, 2016, p. 274). Notre étude, portant sur un nombre plus élevé de francophiles et sur une durée plus longue, valide donc le parrainage comme un outil positif dans l'expérience étudiante sur les plans linguistique, académique et social.

La vision originale du Régime d'immersion en français telle qu'exprimée par son concepteur était d'«amener les deux groupes linguistiques à plus d'échanges via un groupe tampon qui vient de la communauté anglophone mais qui apprécie vivre dans la culture et dans la langue française " (Knoerr, 2016, p. 50). On peut avancer, sur la base de notre étude, qu'une forme de jumelage francophone/francophile du type du "parrainage " peut contribuer à l'intégration académique, sociale et linguistique des étudiants du Régime d'immersion en français et constituer un outil de rapprochement des deux communautés linguistiques et culturelles de l'université.

\section{BIBLIOGRAPHY}

Baudrit, A. (2007a). L'apprentissage collaboratif: plus qu'une méthode pédagogique? Louvain-la-neuve, Belgique : De Boeck.

Baudrit, A. (2007b). Apprentissage coopératif/Apprentissage collaboratif : d'un comparatisme conventionnel à un comparatisme critique. Les Sciences de l'éducation - Pour l'Ère nouvelle, 40(1), 115-136. 
Brown, A. L. et Palincsar, A. S. (1989). Guided cooperative learning and individual knowledge acquisition. Dans L. B. Resnick (dir.), Cognition and Instruction (p. 393-451). Hillsdale, NJ : Lawrence Erlbaum.

Collins, A., Brown, J. S. et Newman, S. E. (1989). Cognitive Apprenticeship : Teaching the craft of reading, writing and mathematics. Dans L. B. Resnick (dir.), Knowing, learning and instruction : Essays in honor of Robert Glaser (p. 453-494). Hillsdale, NJ : Lawrence Erlbaum.

Coulon, A. (1997). Le métier d'étudiant : l'entrée dans la vie universitaire. Paris : PUF.

Cranton, P. (1984). Self-directed and transformative instructional development. Journal of Higher Education, 65(5), 726-744.

De Ketele, J.-M. et Pirot, L. (2000). L'engagement académique de l'étudiant comme facteur de réussite à l'université. Étude exploratoire menée dans deux facultés contrastées. Revue des sciences de l'éducation, 26(2), 367-394.

Demetriou, H., Goalen, P. et Rudduck, J. (2000). Academic performance, transfer, transition and friendship : listening to the student voice. International Journal of Educational Research, 33, 425-441.

Dillenbourg, P. (1999). What do you mean by « collaborative learning »? Dans P. Dillenbourg (dir.), Collaborative learning : Cognitive and computational approaches (p. 1-19). Oxford, Royaume Uni : Pergamon.

Floor, A. (2010). Le parrainage : une nouvelle manière d'intégrer les règles et les valeurs pour éduquer des enfants-citoyens. Analyse UFAPEC, 19(10). Repéré à http://www.ufapec.be/files/files/ analyses/2010/1910-parrainage.pdf

Garrison, D. R. (1995). Constructivism and the role of self-instructional course material : A reply. Distance Education, 16(1), 136-140.

Johnson D. W. et Johnson R. T. (1990). What is cooperative learning? Dans M. Brubacher, R. Payne et K. Rickett (dir.), Perspectives on small group learning (p. 68-80). Oakville, Canada : Rubicon Publishing Inc.

Jonassen, D., Davidson, M., Collins M., Campbell, J. et Haag, B. B. (1995). Constructivism and Computer-Mediated Communication in Distance Education. American Journal of Distance Education, $9(2), 7-26$.

Knoerr, H. (2010). L'immersion au niveau universitaire : nouveaux modèles, nouveaux défis, nouvelles stratégies. Cahiers de l'ILOB/ OLBI Working Papers, 1(1), 89-110.

Knoerr, H. (2016). Interprétations politiques et aménagements institutionnels pour l'immersion à l'Université d'Ottawa. Dans Knoerr, H., Weinberg, A. et Gohard-Radenkovic, A. (dir.), L'immersion française à l'université : Politiques et pédagogies (p. 45-76). Ottawa, Canada : PUO.

Knoerr, H. et Weinberg, A. (2013). L'immersion à l'Université d'Ottawa : une innovation héritée du passé. Recherches en didactique des langues et des cultures : Les Cahiers de l'Acedle, 10(3), 15-35.

Lamoureux, S. (2013). L'expérience étudiante au Régime d'immersion en français : perspectives et constats. Cahiers de l'ILOB, 6, 109-121. DOI :10.18192/olbiwp.v6i0.1134

Lave, J. et Wenger, E. (1991). Situated Learning : Legitimate Peripheral Participation. Cambridge, Royaume Uni : Cambridge University Press.

Lebow, D. (1991). Constructivist values for instructional systems design: Five principles toward a new mindset. Educational Technology Research et Development, 41(3), 4-16. 
Michaut, C. (2002). L'efficacité des dispositifs d'aide aux étudiants dans les universités. Recherche et Formation, 43, 101-113.

Pléty, R. (1996). L'apprentissage coopérant. Lyon, France : Presses universitaires de Lyon. Romainville, M. (2000). L'échec dans l'université de masse. Paris, France : L'Harmattan

Procidiano, M. E. et Heller, K. (1983). Measures of perceived social support from friends and from family : Three validation studies. American Journal of Community Psychology, 11, 1-24.

Sarason, I. G., Sarason, B. R., Potter III, E. H. et Antoni, M. H. (1985). Life events, social support, and illness. Psychosomatic Medicine, 47(2), 156-163.

Sarason, I. G., Levine, H. M., Basham, R. B. et Sarason, B. R. (1983). Assessing social support : The Social Support Questionnaire. Journal of Personality et Social Psychology, 44(1), 127-139.

Séror, J. et Lamoureux, S. (2014). Intégrer les étudiants anglophones dans le cadre d'un programme d'immersion universitaire au Canada. Dossiers des sciences de l'éducation, 32, 95-110.

Springer, C. (2010). La dimension sociale dans le CECR : pistes pour scénariser, évaluer et valoriser l'apprentissage collaboratif. Canadian Modern Language Review / La revue canadienne des langues vivantes, 66(4), 511-523.

von Glasersfeld, E. (1995). A constructivist approach to teaching. Dans L. P. Steffe et J. Gale (dir.), Constructivism in education (p. 3-16). Hillsdale, NJ : Erlbaum.

Vygotski, L. S. (1962). Thought and language. Cambridge, MA : M.I.T. Press.

Weinberg, A. (2013). Parrainage francophiles-francophones à l'Université d'Ottawa dans le cadre du Régime d'immersion : pratique et perceptions. Dans Yasri-Labrique, E. (dir.), Didactique contrastive : questionnements et applications (p. 105-117). Montpellier, France : Éditions Cladole.

Weinberg, A., Knoerr, H. et Gohard-Radenkovic, A. (2016). Pratiques collaboratives entre les différents acteurs de l'immersion : mentorat, parrainage, tutorat, tandem. Dans H. Knoerr, A. Weinberg et A. Gohard-Radenkovic (dir.), L'immersion française à l'université : Politiques et pédagogies (p. 253-282). Ottawa, Canada : PUO.

Wilson, B. et Cole, P. (1991). A review of cognitive teaching models. Educational Technology Research \& Development, 39(4), 47-64.

\section{NOTES}

1. Pour une description complète du Régime d'immersion en français, voir Knoerr et Weinberg (2013).

2. La notion d'expérience étudiante, dans la ligne de mire des universités nord-américaines depuis quelques années, est définie par Lamoureux (2013) comme «L'expérience étudiante ne se limite pas aux activités scolaires mais compte également la dimension sociale, qui comprend les interactions avec les autres étudiants sur le campus et ailleurs, ainsi que les interactions avec l'environnement citadin » (p. 118).

3. Les professeurs de discipline doivent gérer dans leurs cours de première année plusieurs centaines d'étudiants et des assistants d'enseignement, ils ne sont donc pas enclins à alourdir davantage leur tâche.

4. Pour des questions de déontologie, il n'a pas été possible de faire remplir le questionnaires aux étudiants francophones. 
5. Au Canada l'enseignement du français langue seconde est offert selon diverses modalités, allant du français cadre, où le français est enseigné comme matière, jusqu'à l'immersion, où un nombre variable de matières sont enseignées en français.

\section{ABSTRACTS}

en

This study reports on a program pairing up first-year Anglophone students registered in a French immersion stream with Francophone students enrolled in the same discipline courses delivered in French in a bilingual Canadian university. This program has three main goals : (1) improve immersion students' academic success; (2) facilitate their integration in the student community; (3) improve their French language skills. This collaborative work program was offered during three thirteen week- long terms over two academic years and assessed via Likerttype questionnaires completed by the participants. Although the number of respondents was relatively limited, results indicate that students view the program as a way to improve their language and academic skills and to facilitate their integration into the student community. The program also seems to foster closer ties between the two linguistic and cultural communities.

fr

Cette étude porte sur un programme de parrainage offrant aux étudiants anglophones de première année inscrits dans une filière d'immersion universitaire en français dans une université canadienne bilingue la possibilité d'être associés à des étudiants francophones qui suivent le même cours de discipline en français. Le but poursuivi est triple : (1) améliorer leur réussite académique; (2) favoriser leur intégration sociale dans la communauté étudiante; (3) améliorer leurs compétences linguistiques en français. Nous avons proposé ce programme de travail collaboratif au cours de trois sessions universitaires de treize semaines chacune échelonnées sur deux ans et nous l'avons évalué au moyen d'un questionnaire de type Likert remplis par les participants. Malgré le nombre relativement réduit des répondants, nous pouvons conclure que les étudiants perçoivent le parrainage comme un moyen d'améliorer leurs compétences linguistiques et académiques et de favoriser leur intégration sociale. De plus, il semble permettre un rapprochement entre les deux communautés linguistiques et culturelles.

\section{INDEX}

Mots-clés: parrainage, immersion universitaire, français langue seconde, apprentissage collaboratif, transition postsecondaire

\section{AUTHOR}

\section{HÉLÈNE KNOERR}

Université d'Ottawa, hknoerr@uottawa.ca 